\title{
Holographic spectrum splitter for ultra-high efficiency photovoltaics
}

Sunita Darbe, Matthew D. Escarra, Emily C. Warmann, Harry A. Atwater

Sunita Darbe, Matthew D. Escarra, Emily C. Warmann, Harry A. Atwater, "Holographic spectrum splitter for ultra-high efficiency photovoltaics," Proc. SPIE 8821, High and Low Concentrator Systems for Solar Electric Applications VIII, 882105 (9 September 2013); doi: 10.1117/12.2024610

EviE Event: SPIE Solar Energy + Technology, 2013, San Diego, California, United States 


\title{
Holographic spectrum splitter for ultra-high efficiency photovoltaics \\ Sunita Darbe ${ }^{1}$, Matthew D. Escarra ${ }^{1,2, *}$, Emily C. Warmann ${ }^{1}$, Harry A. Atwater ${ }^{1}$ \\ ${ }^{1}$ Thomas J. Watson Laboratory of Applied Physics, California Institute of Technology, Pasadena, CA USA 91125 \\ ${ }^{2}$ Department of Physics and Engineering Physics, Tulane University, New Orleans, LA 70118, USA
}

\begin{abstract}
To move beyond the efficiency limits of single-junction solar cells, junctions of different bandgaps must be used to avoid losses from lack of absorption of low energy photons and energy lost as excited carriers thermalize to the semiconductor band edge. Traditional tandem multijunction solar cells are limited, however, by lattice-matching and current-matching constraints. As an alternative we propose a lateral multijunction design in which a compound holographic optic splits the solar spectrum into four frequency bands each incident on a dual-junction, III-V tandem cell with bandgaps matched to the spectral band. The compound splitting element is composed of four stacks of three volume phase holographic diffraction gratings. Each stack of three diffracts three bands and allows a fourth to pass straight through to a cell placed beneath the stack, with each of the three gratings in the stack responsible for diffracting one frequency band.

Generalized coupled wave analysis is used to model the holographic splitting. Concentration is achieved using compound parabolic trough concentrators. An iterative design process includes updating the ideal bandgaps of the four dual-junction cells to account for photon misallocation after design of the optic. Simulation predicts a two-terminal efficiency of $36.14 \%$ with $380 x$ concentration including realistic losses.
\end{abstract}

Keywords: spectrum splitting, holographic optical element, photovoltaics, photonic design, III-V semiconductor material

\section{INTRODUCTION}

Single-junction photovoltaics have a theoretical detailed-balance efficiency limit of about $33 \%{ }^{1}$. A great deal of research and development have led to crystalline silicon and GaAs cells which approach this thermodynamic limit with record efficiencies of $25.0 \%$ and $28.8 \%$ respectively ${ }^{2}$. To increase photovoltaic conversion efficiencies beyond this, we turn to multijunction solar cells, which address losses due to lack of absorption of photons with energy below the material's bandgap energy and also address losses due to thermalization of carriers generated by photons with energy greater than the bandgap energy. Together these two losses add up to over $40 \%$ of total incident solar power ${ }^{3}$.

The higher bandgap cells must generate a higher collection voltage for the splitting to be worthwhile. For high-quality semiconductor materials the $\mathrm{V}_{\mathrm{OC}}$ of the cell is almost linearly related to the bandgap of the semiconductor material ${ }^{4}$. Thus using higher bandgap materials to collect higher energy photons returns more electrical energy upon absorption and collection. This motivates incorporation of many, high quality absorber materials into a photovoltaic conversion system. The III-V alloy system provides direct bandgap materials of high material quality with bandgap tunability over much of the target range of interest for solar applications, so we focus on this material system.

* escarra@tulane.edu; $\quad$ phone +1(504)-862-8673; $\quad$ fax +1(504)-862-8702; $\quad$ escarra.tulane.edu

High and Low Concentrator Systems for Solar Electric Applications VIII, edited by Adam P. Plesniak, Proc. of SPIE Vol. 8821, 882105 · C 2013 SPIE · CCC code: 0277-786X/13/\$18 · doi: 10.1117/12.2024610 
Many methods have been explored for incorporating many absorbers into photovoltaic devices. In the past decade, epitaxially grown, monolithic tandem cells (typically 2-3 absorbers, or junctions, and as many as 5) have been the focus of research and development. This kind of cell has the advantage of intrinsic splitting of the solar spectrum into different frequency bands. Each cell acts as a long-pass filter allowing lower energy, unabsorbed photons to pass through to the next cell. However since the lattice constants of epitaxially grown layers must be the same or similar to maintain high material quality and minimize defects, there are limits to the number of absorber materials that can be incorporated. Additionally, since monolithic tandem cells are electrically in series, each junction is limited by the current generated by the cell generating the least current. These cells are designed so that this current-matching condition maximizes current for a particular solar spectrum. As the solar input varies over the course of a day or year or with changing location, the current match may no longer hold, decreasing efficiency. For these reasons, we are exploring "lateral" spectral-splitting options in which solar cells are electrically and optically in parallel. To achieve optically in parallel operation, spectralsplitting optics are necessary, where before the upper cells acted as a filter for lower cells. This spectral-splitting optic also allows each cell to act electrically independently, enhancing annual energy production.

Recent efforts have used diffraction ${ }^{5}$, interference-based filtering ${ }^{6}$, refraction ${ }^{7}$, specular reflection ${ }^{8}$, and diffuse reflection $^{9}$ to split the solar spectrum. Imenes et al. has reviewed much of the previous work ${ }^{10}$. Groups have also worked on holographic approaches ${ }^{11,12}$. The efficiencies of lateral multijunction devices, however, still lags behind those of traditional multijunction cells and devices. Our work aims to experimentally demonstrate the incorporation of 8 junctions through holographic spectrum-splitting to reach ultra-high efficiency ${ }^{13}$.

Among diffractive optics available, holograms have the advantage of avoiding complex lithographic fabrication steps. Hologram fabrication (using the exposure of a recording material to an interference pattern between coherent light sources) allows large-area fidelity of recording, creating a low-scatter, high-performance diffractive optic. Volume phase holograms have thicknesses much larger than their fringe spacings, which leads to volume effects becoming important ${ }^{14}$. They can have diffraction efficiencies (intensity of total incident light to intensity of light going into the correct diffracted order) of up to $100 \%$ with low-absorption, low-scatter materials.

The holographic material is a key component of this design. We require low absorption and scattering over a broad wavelength range $(300 \mathrm{~nm}-1700 \mathrm{~nm})$, high resolution, tunable properties, high diffraction efficiencies and ease of processing. In addition to all this, incorporation into a solar application requires a long lifetime ( $>25$ years), the ability to withstand high-intensity light without performance degradation, and resistance to the elements and to breakage. These criteria make dichromated gelatin (DCG) the top choice with its low absorption and scattering and a wide range of index of refraction modulation $(\Delta \mathrm{n})$. Common applications of DCG holograms include laser applications such as pulse compression, beam-splitting and beam-combining which require high light intensity exposure. DCG is hygroscopic and thus requires encapsulation. Additionally, the index of refraction modulation can vary from 0.01 to up to 0.4 , but as this index modulation increases scattering into spurious diffraction orders also increases ${ }^{14}$, so we have restricted the range of search from 0.01 to 0.06 . Layers can be easily deposited and exposed at thicknesses less than $30 \mu \mathrm{m}$. For a given value of $\Delta \mathrm{n}$ there is a minimum effective thickness to get high diffraction efficiency. Thicker gratings and lower $\Delta \mathrm{n}$ give lower bandwidth diffraction peaks and likewise thinner gratings with higher $\Delta \mathrm{n}$ give higher bandwidth peaks.

\section{DESIGN AND METHODS}

\subsection{Our design}

The holographic spectrum splitter is a linear compound element composed of four stacks of three gratings each arranged in a line. Each stack diffracts normal or nearly normal incidence light into four spectral bands. Details of the spectral bands are specified in Table 1. Each of the three diffraction gratings diffracts one spectral band into its first diffracted order toward the cell it is intended for and a fourth band of light passes straight through the three holographic gratings (in their zeroth order) to the cell directly underneath. Each grating stack sends the highest energy light incident on the stack toward the tandem cell designed for high-energy photons, and the lowest energy light incident upon it toward the rightmost cell, designed for low-energy photons. The highest energy tandem has subcells of bandgaps $2.25 \mathrm{eV}$ and 1.84 $\mathrm{eV}$, the second highest energy tandem $1.60 \mathrm{eV}$ and $1.42 \mathrm{eV}$, the third highest $1.23 \mathrm{eV}$ and $1.06 \mathrm{eV}$, and the lowest energy tandem 0.93 and $0.74 \mathrm{eV}$. Lattice-matched III-V alloys can be found for each of these subcell pairs. Edge effects in the holograms are assumed to be negligible. 
Table 1. Wavelength range of spectral bands.

\begin{tabular}{|c|c|c|}
\hline Band & $\begin{array}{c}\text { Design } \boldsymbol{\lambda} \\
\text { (nm) }\end{array}$ & $\begin{array}{c}\text { Bandwidth } \\
\text { (nm) }\end{array}$ \\
\hline 1 & 437 & $300-674$ \\
\hline 2 & 700 & $675-873$ \\
\hline 3 & 970 & $874-1170$ \\
\hline 4 & 1425 & $1171-1676$ \\
\hline
\end{tabular}

Each grating is recorded on a substrate. During post-processing a top glass layer is placed on top and the edges are sealed with a moisture barrier for full encapsulation. The effective index of refraction of the DCG gratings is 1.3 while the substrate, commonly fused silica or glass ranges from 1.45 to 1.55. The index of DCG during recording (before development), however, is 1.55 . It is desirable to have an index-matched substrate during the hologram fabrication to avoid artifacts due to Fresnel reflections off the substrate during the recording process. Alternatively, having an index match during use in the grating stack reduces Fresnel reflections during the lifetime of the grating stack. This trade-off also incentivizes the use of holographic materials which can be better index-matched to available substrates and which do not require post-processing which might alter their pre- and post-recording properties.

In order to get a realistic system efficiency from the spectrum splitting system, we use various estimates to account for losses. For the cells, a detailed balance efficiency is adjusted by assuming that only $90 \%$ of incident photons are absorbed and that the active materials have 1\% external radiative efficiency (ERE). These de-rating factors account for losses such as non-radiative recombination and parasitic absorption and produce realistic cell efficiency estimates from the theoretical detailed balance calculation. They have a combined de-rated detailed balance efficiency of $46.97 \%$ using these de-rated parameters of $90 \%$ absorption, 1\% ERE for unconcentrated illumination and perfect spectral splitting. With a concentration of $100 x$ this goes to $52.7 \%$. The figure of merit for the splitting performance is the optical efficiency, defined as

$$
\eta_{\text {optical }}=\frac{\text { System power with actual splitting }}{\text { Power with perfect splitting }},
$$

where system power refers to the power obtained by independently connecting the four dual-junction cells and using DC-to-DC converters to combine the output current and voltage into a two-terminal output.

\subsection{Design considerations}

Each grating is designed for a particular wavelength within its spectral band. Holographic diffraction gratings have a decrease in diffraction efficiency as the wavelength deviates from this design wavelength as shown in Figure 1. We aim to have the full width, half maximum of each diffraction peak equal to the desired bandwidth to get optimal diffraction of each band and minimize cross-talk between spectral bands.

Only the light at the design wavelength of a given grating will get diffracted to the correct angle. As the wavelength deviates slightly from the design wavelength, so too does the angle corresponding to the output diffraction order shift slightly. As the wavelength increases the diffraction angle increases. Thus in the spectral band in which $874 \mathrm{~nm}$ to 1170 $\mathrm{nm}$ light is to be diffracted 10 degrees, the $970 \mathrm{~nm}$ light will go 10 degrees, the $874 \mathrm{~nm}$ light will go $<10$ degrees and the $1170 \mathrm{~nm}$ light will go $>10$ degrees. Thus most of the light is falling not just on the intended cell, but also onto one of its neighbors. Photons falling on cells with bandgaps to the blue of their energy will not be absorbed at all while photons falling on cells with bandgap to the red of their energy can get collected and generate some energy. Additionally the more energetic spectral band contains the most power, so it is most important that this band get to the correct cell. The extended structure of the array is a head-to-head, tail-to-tail arrangement, to minimize photons going to cells of completely different bandgaps. This dependence of output angle on wavelength and this extended geometry are accounted for in our holographic simulations. 


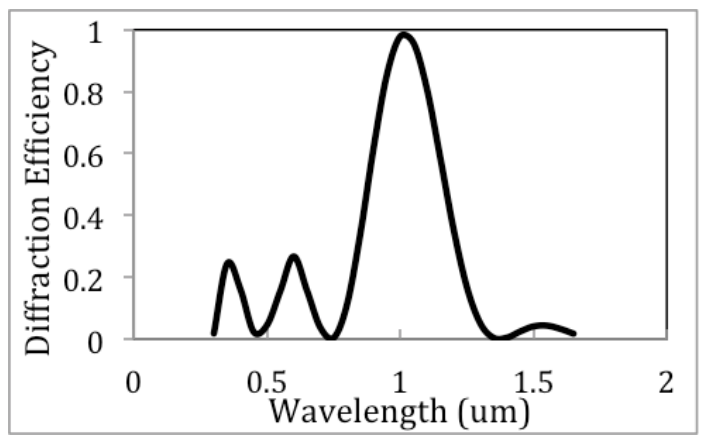

Figure 1. Diffraction efficiency vs. wavelength for a grating designed for spectral band 3, which has a central wavelength of 970 $\mathrm{nm}$ and a bandwidth of $296 \mathrm{~nm}$. As the wavelength deviates from the design wavelength, diffraction efficiency goes down. Higher order harmonics are also present.

The holograms are sensitive to the angle of incidence of light, and this sensitivity is increased when stacking holograms, which act in concert. Thus, they must be incorporated into a tracker. The submodule performance drops off significantly for light incident at a deviation of greater than $2^{\circ}$ from normal. This angular sensitivity is similar to that of highconcentration optics. Since using angle-of-incidence sensitive diffractive optics requires tracking of the sun and use of only the light in the direct solar spectrum rather than the global solar spectrum, concentration allows both a compensation for the diffuse light lost as well as the potential to access much higher overall efficiencies.

Increasing concentration, holding all else constant, improves efficiency. Non-imaging optical elements allow concentration that can reach thermodynamic limits ${ }^{15}$. A compound parabolic concentrator (CPC) takes any light incident on its input aperture within a certain half-angle (its acceptance angle) from the normal and reflects it to its output aperture. In the concentration scheme used for the holographic splitter (Fig. 2), the top CPC is a curved, silvered mirror, which concentrates light orthogonal to the direction of spectral splitting. The secondary CPC is concentrating in two directions with rectangular input and output apertures. It is solid and made of a high-index polymer $(n=1.65)$ giving an $n^{2}$ enhancement in the concentration relative to a hollow CPC with the same acceptance angle. The reflection at the surface of the CPC is due to total-internal reflection at the polymer-air interface. The rectangular shape comes from intersecting two trough CPC profiles. The inset shows the shape of the secondary concentrator. The corners add some loss relative to a trough that concentrates in only one direction. The optimum output to the cells accounting for both increased concentration and increased loss from the concentrator must be balanced.

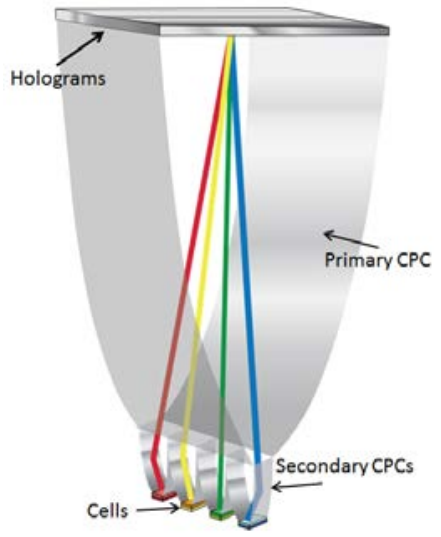

Figure 2. Schematic of holographic splitter showing two-axis concentration using a hollow trough compound parabolic concentrator orthogonal to the direction of splitting immediately below the holograms followed by filled troughs concentrating both in the direction of splitting and orthogonal to it.

The holographic spectrum splitter assumes four equally-sized tandem solar cells. Without concentration, the holograms and the solar cells are the same size. Increasing concentration allows smaller active device areas and thus lower cell costs, though it adds on costs associated with the concentration. 


\subsection{Hologram Modeling}

We use Moharam and Gaylord's 1977 generalized coupled wave analysis (GCWA) to model the holographic gratings ${ }^{16}$. GCWA neglects second derivatives of the electric field associated with each diffracted order (a slowly varying field approximation). Additionally, reflected diffracted orders are neglected. This leaves a system of $1^{\text {st }}$ order, coupled linear differential equations to solve. This method gives the diffracted intensity in those output modes that are retained in the calculation. In our calculations, transmitted diffracted orders -7 to +7 have been retained. This large number has been retained due to diffraction into progressively higher orders as the initially normally incident solar light passes through each grating stack.

The GCWA approach balances accuracy and computational expense better than more conventional choices. Coupledwave analysis, considering only the input $\left(0^{\text {th }}\right.$ order $)$ and $1^{\text {st }}$ order output is a valid approximation when the angle of incidence is near the Bragg angle and the grating is thick. In our case of stacked gratings and very broadband illumination, there will be much incident light that is far from the Bragg condition and so we need a broader theoretical formulation to consider diffraction from our gratings. Rigorous coupled wave analysis, on the other hand, gives a more accurate solution (it is exact for a grating of infinite area when an infinite number of diffracted orders are used) but is computationally expensive.

\section{DATA/RESULTS}

To model the full compound holographic spectrum splitter, the output of each successive grating in a particular stack is found using GCWA for normally incident light. The intensity of light diffracted into orders -7 to +7 by the top hologram is calculated and those orders with greater than $0.01 \%$ diffraction efficiency are retained and become an input into the $2^{\text {nd }}$ grating in the stack. Similarly the output of the $2^{\text {nd }}$ grating becomes the input into the third grating. Finally, the output intensities and diffraction angles from the final grating are used to determine which underlying solar cell any particular output from the bottommost grating will hit. The total output fraction of input light intensity hitting each cell can be converted to a photon flux using the AM1.5d spectrum to determine how many above bandgap photons are hitting each of the four tandem cells.

The flux hitting each cell becomes the input to detailed balance calculations, which give a conversion efficiency for the sub-module. The grating model accounts for any misallocated photons due to the optics. The parameters of the holographic spectral splitter grating are given in Table 2. The index of refraction variation $\Delta \mathrm{n}=0.015$ is used for all of the gratings. Recognizing that the optimal current matched tandem cells for the actual spectral bands generated from the splitting optics will not be the same as the best bandgaps for perfect splitting, the bandgap selection is re-optimized. This gives bandgaps of $2.24 \mathrm{eV} / 1.38 \mathrm{eV}$ for the top cell, $1.74 \mathrm{eV} / 1.12 \mathrm{eV}$ for the second highest, $1.36 \mathrm{eV} / 0.94 \mathrm{eV}$ for the third tandem, and $1.06 \mathrm{eV} / 0.75 \mathrm{eV}$ for the lowest energy tandem. These pairs are current-matched but not lattice-matched. Including realistic cell performance with $90 \%$ absorption and $1 \%$ external radiative efficiency de-rating factors at the cell level and the splitting of the holographic stacks, the total system efficiency with the re-optimized bandgaps and 380x concentration is found to be $43.19 \%$. The optical efficiency of these holograms is found to be $78.80 \%$. A $5 \%$ loss due to Fresnel reflections between the gratings and their substrates, off the front face of the cells, and from the interface between the two CPC stages is assumed. A $2 \%$ series resistance due to electrical contacts and an additional $2 \%$ due to power conditioning electronics are assumed ${ }^{17}$. Finally the losses due to the concentrators are estimated to be $8.3 \%$. All together the sub-module is expected to have a realistic efficiency of $36.14 \%$. 
Table 2. Holographic spectral splitter design parameters.

\begin{tabular}{|c|c|c|c|c|}
\hline Stack & $\begin{array}{c}\text { Design } \boldsymbol{\lambda} \\
(\mathbf{n m})\end{array}$ & $\begin{array}{c}\text { Phi } \\
\mathbf{(}^{\circ} \mathbf{)}\end{array}$ & $\begin{array}{c}\mathbf{L} \\
(\text { lines/mm) }\end{array}$ & $\begin{array}{c}\mathbf{d} \\
(\boldsymbol{\mu m})\end{array}$ \\
\hline \multirow{3}{*}{$\mathbf{1}$} & 1425 & -76.98 & 411.1 & 22 \\
\cline { 2 - 5 } & 970 & -80.56 & 439.4 & 22 \\
\cline { 2 - 5 } & 700 & -85.00 & 323.7 & 22 \\
\hline \multirow{3}{*}{$\mathbf{4}$} & 437 & 85.00 & 518.5 & 14.5 \\
\cline { 2 - 5 } & 970 & -85.00 & 233.6 & 22 \\
\cline { 2 - 5 } & 1425 & -80.56 & 299.1 & 22 \\
\hline \multirow{3}{*}{3} & 437 & 80.56 & 975.4 & 14.5 \\
\cline { 2 - 5 } & 700 & 85.00 & 323.7 & 22 \\
\cline { 2 - 5 } & 1425 & -85.00 & 159.0 & 22 \\
\hline \multirow{3}{*}{4} & 437 & 76.98 & 1340.7 & 14.5 \\
\cline { 2 - 5 } & 700 & 80.56 & 608.9 & 22 \\
\cline { 2 - 5 } & 970 & 85.00 & 233.6 & 22 \\
\hline
\end{tabular}

\section{CONCLUSIONS}

We have designed a holographic spectrum splitter than generates four spectral bands and separates them onto four dualjunction solar cells for a total of 8 bandgaps. The system is designed using commercially available simple sinusoidal holographic diffraction gratings. Estimated system efficiency accounting for realistic cell performance and other losses is $36.14 \%$, matching current records for lateral spectral splitting, with the potential for much higher efficiency upon future design iteration.

\section{ACKNOWLEDGEMENTS}

The information, data, or work presented herein was funded in part by the Advanced Research Projects Agency-Energy (ARPA-E), U.S. Department of Energy, under Award Number DE-AR0000333. The authors acknowledge the Dow Chemical Company for funding. Thanks to E. Kosten for useful discussion.

\section{REFERENCES}

[1] Nelson, J., [The Physics of Solar Cells], Imperial College Press, London, 31-35 (2003).

[2] Green, M. A., Emery, K., Hishikawa, Y., Warta, W. and Dunlop, E. D., "Solar cell efficiency tables (version 41)," Prog. Photovolt: Res. Appl. 21(1), 1-11 (2013).

[3] Polman, A. and Atwater, H. A., "Photonic design principles for ultrahigh-efficiency photovoltaics," Nat. Mat. 11(3), 174-177 (2012).

[4] King, R. R., Bhusari, D., Boca, A., Larrabee, D., Liu, X.-Q., Hong, W., Fetzer, C. M., Law, D. C. and Karam, N. H., "Band gap-voltage offset and energy production in next-generation multijunction solar cells," Prog. Photovolt.: Res.

Appl. 19(7), 797-812 (2011).

[5] Kim, G., Dominguez-Caballero, J.-A., Lee, H., Friedman, D. and Menon, R., "Increased photovoltaic power output via diffractive spectrum separation," Phys. Rev. Lett. 110(12), 123901 (2013).

[6] Wang, X., Waite, N., Murcia, P., Emery, K., Steiner, M., Kiamilev, F., Goossen, K., Honsberg, C. and Barnett, A., "Lateral spectrum splitting concentrator photovoltaics: direct measurement of component and submodule efficiency," Prog. Photovolt.: Res. Appl. 20(2), 149-165 (2012).

[7] Stefancich, M., Zayan, A., Chiesa, M., Rampino, S., Roncati, D., Kimerling, L. and Michel, J., "Single element spectral splitting solar concentrator for multiple cells CPV system,” Opt. Express 20(8), 9004-9018 (2012).

[8] Mitchell, B., Peharz, G., Siefer, G., Peters, M., Gandy, T., Goldschmidt, J. C., Benick, J., Glunz, S. W., Bett, A. W. and Dimroth, F., "Four-junction spectral beam-splitting photovoltaic receiver with high optical efficiency," Prog. 
Photovolt.: Res. Appl. 19(1), 61-72 (2011).

[9] Goldschmidt, J. C., Do, C., Peters, M. and Goetzberger, A., "Spectral splitting module geometry that utilizes light trapping,” Sol. Energy Mat. Sol. Cells 108, 57-64 (2013).

[10] Imenes, A. G. and Mills, D. R., "Spectral beam splitting technology for increased conversion efficiency in solar concentrating systems,” Sol. Energy Mat. Sol. Cells 84(1-4), 19-69 (2004).

[11] Zhang, D., Gordon, M., Russo, J. M., Vorndran, S., Escarra, M., Atwater, H. A. and Kostuk, R. K., "Reflection hologram solar spectrum-splitting filters,” Proc, SPIE 8468, (2012).

[12] Torrey, E. R., Ruden, P. P. and Cohen, P. I., "Performance of a split-spectrum photovoltaic device operating under time varying spectral conditions,” J. Appl. Phys. 109(7), 074909 (2011).

[13] Escarra, M. D., Darbe, S., Warmann, E. C. and Atwater, H. A., "Spectral-Splitting Photovoltaics: Holographic Spectrum Splitting in Eight-Junction, Ultra-High Efficiency Module” Proc. IEEE PVSC 39 (2013).

[14] Hariharan, P., [Optical Holography: Principles, techniques and applications], Cambridge University Press, Cambridge \& New York, 45-65, 107-111 (1996).

[15] Winston, R., "Light Collection within the Framework of Geometrical Optics,” J. Opt. Soc. Am. 60(2), 245-247 (1970).

[16]Magnusson, R. and Gaylord, T. K., “Analysis of multiwave diffraction of thick gratings,” J. Opt. Soc. Am. 67(9), 1165-1170 (1977).

[17] Agamy, M. S., Chi, S., Elasser, A., Harfman-Todorovic, M., Jiang, Y., Mueller, F. and Tao, F., “A High-PowerDensity DC-DC Converter for Distributed PV Architectures,” IEEE J. Photovolt. 3(2), 791-798 (2013). 